\title{
EFFECT OF DC ELECTRIC FIELDS ON FLAME SPREAD OVER TWIN ELECTRICAL WIRES
}

\author{
Jeong Park ${ }^{1^{*}}$, Sun Ho Park ${ }^{1}$, Min Suk Cha ${ }^{2}$, Suk Ho Chung ${ }^{2}$ \\ ${ }^{1}$ Department of Mechanical Engineering, Pukyong National University, Busan, Korea \\ ${ }^{2}$ Clean Combustion Research Center, King Abdullah University of Science and Technology (KAUST), \\ Thuwal, Saudi Arabi
}

\begin{abstract}
The effect of DC electric field on the behaviors of spreading flame over polyethylene (PE)-insulated twin electrical wires was investigated by varying the wire gap $(S)$ and voltage $\left(V_{D C}\right)$. The twin flame spreads with the same flame spread rate (FSR) independently when no electric field is applied. With an applied DC electric field, the twin flame interacts such that FSR, flame width, and the leaning direction of interacting twin flames vary appreciably. The spread rate for wire flame with negative voltage $(S F-)$ was larger than that with positive voltage ( $S F+)$ during transient period and then the two became the same in a developed region (a quasi-steady spread). Such a flame behavior could be grouped into two: twin flame spread (regime I) and single flame spread (regime II) after the extinction of SF+. Each regime could be categorized into three sub-regimes depending on $S$ and $V_{D C}$. For small $V_{D C}$, the flame leaned toward the burnt wire, reducing FSR. With further increasing $V_{D C}, F S R$ increased due to the ionic wind effect and then decreased via the mass loss of molten PE. These non-monotonic behavior of FSR with DC voltage can be attributed the behaviors of molten PE, exhibiting dripping, electrospray, and di-electrophoresis phenomena. For further increased voltage, the flames were extinguished by streamer generation and an electrical short occurred at excessive voltages.
\end{abstract}

Keywords: Electrical wire; DC electric field; Spread rate; Electrospray.

\section{INTRODUCTION}

An electrical wire fire could occur due to unexpected arcing, short circuit, and/or overheating of wire, leading to ignition of flammable materials and subsequently a spread of fire. Electrical wire fires are one of the main causes of fires in buildings and households [1-3], as well as in closed environments such as aircraft and spacecraft. In this regard, the fundamental characteristics of wire fires have been studied extensively, considering many parameters such as the insulation material, insulator thickness, gravity level, ambient flow and pressure [5-10], typically by adopting a polyethylene (PE) insulated $\mathrm{Ni}-\mathrm{Cr}$ wire as a model problem.

When an electrical wire ignited after electrical short, the wire can still be under the influence of an electric field as an open circuit. In such a case, charged particles generated through chemi-ionization and subsequent ion chemistry in the reaction zone of a spreading flame can be influenced by the electric field through the Lorentz force [12].

A limited number of studies on the effect of electric fields on spreading flame over electrical wires has been conducted [13-15], which were focused on a flame spread for a single wire. The present work investigates the effect of applied DC electric fields on flame spread over twin electrical wires, focusing on the interaction between the two spreading flames. Flame spread rates are reported along with several interesting phenomena such as flame extinction, molten PE dripping, electrospray and di-electrophoresis phenomena, streamer generation, and electrical short.

\section{EXPERIMENT}


The apparatus consisted of twin electrical-wires and wire-holder, electrical power supplies, and a visualization setup, as schematically shown in Fig. 1. PE-insulated electrical wire with $0.8 \mathrm{~mm}$ in diameter $(0.5 \mathrm{~mm}$ nichrome core diameter) and $216 \mathrm{~mm}$ in length was used. The initial $70 \mathrm{~mm}$ from one end of the wire and the final $10 \mathrm{~mm}$ were excluded due to the ignition transient and to avoid interaction with the wire holder, leaving $136 \mathrm{~mm}$ of wire length available for flame spread. The twin wires were installed horizontally on a wire holder (with holes at $2 \mathrm{~mm}$ intervals), made of non-conductive acetal resin. For each wire, one end was attached to a fixture and the other end was connected to a spring to prevent bending due to thermal expansion during flame spread. The twin wire and holder were surrounded by acetal mesh screen to prevent outside disturbances.

Twin flames were initiated by two hot-wire igniters $(1 \mathrm{~mm}$ in diameter, $\mathrm{Rh} / \mathrm{Pt} 13 \%)$, which was placed on two air cylinders. To minimize interactions between the ignition system and applied DC electric fields, the igniters were restored to their original positions away from each wire after ignition. A programmable logic controller was used to control the time sequence of the experiment. Three video cameras were triggered to capture images of flame spread, and the recorded images were analyzed using a Matlab-based code. Two DC power supplies were used to apply positive and negative electric voltages to obtain a large potential difference between the two wires. The applied voltage $\left( \pm V_{\mathrm{DC}}\right)$ was varied over the range $0-10 \mathrm{kV}$ and monitored using an oscilloscope. The wire gap $(S)$ between the two wires varied from 11 to $61 \mathrm{~mm}$ with the interval of $10 \mathrm{~mm}$.

\section{RESULTS AND DISCUSSION Overall behavior of flame spread}

Figure 2 shows images of spreading flames with applied DC voltage $\left(V_{\mathrm{DC}}\right)$ for the wire gap $S=51 \mathrm{~mm}$, along with the baseline case with no applied electric filed. For the baseline case, the flame spreads in parallel and are nearly vertically. For $V_{\mathrm{DC}}= \pm 1 \mathrm{kV}$, the spreading flame with positive (negative) voltage (hereafter $\mathrm{SF}+(\mathrm{SF}-)$ ) leans toward the burnt wire. When the electric fields becomes sufficiently strong at $\pm 2.5 \mathrm{kV}, \mathrm{SF}+$ tilts toward $\mathrm{SF}-$ and thus leans toward the unburned wire, an indication of the interaction of two flames. While the size of SF- becomes larger than that at $\pm 1 \mathrm{kV}$ and the molten PE of SF- drips onto the ground. For $\pm 3.5 \mathrm{kV}, \mathrm{SF}+$ leans toward SF-. The size of SFbecomes smaller and a series of droplets ejected from molten PE surface, as marked with red circle, which was attributed to the electrospray phenomenon [15]. A small droplet is detached from the main body of molten PE and moved toward the burnt wire, which was attributed to di-electrophoresis phenomenon [18]. Subsequently fine droplets also eject from the detached droplet surface due to electrospray phenomenon. From the top view at $V_{\mathrm{DC}} \leq \pm 3.5 \mathrm{kV}$, $\mathrm{SF}+$ is always tilted toward $\mathrm{SF}-$, while $\mathrm{SF}-$ fluctuates unstably.

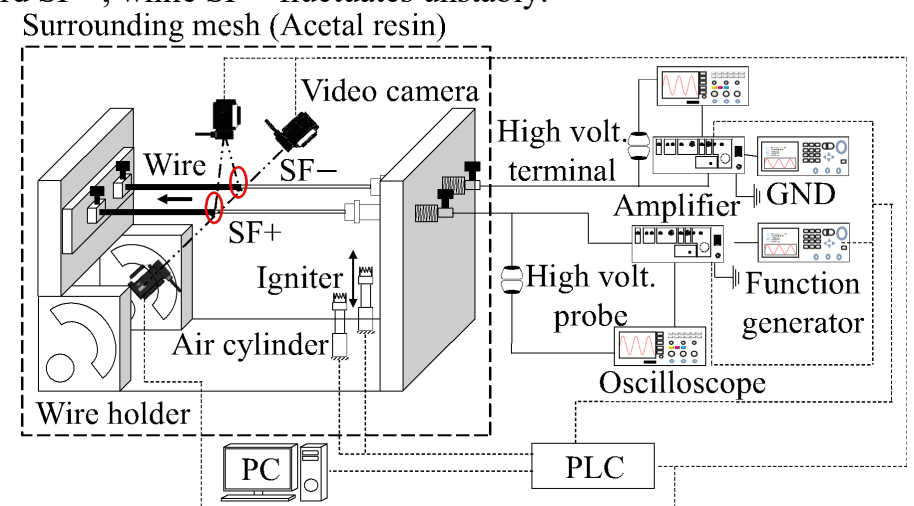

FIGURE 1: Schematic of experimental setup. 


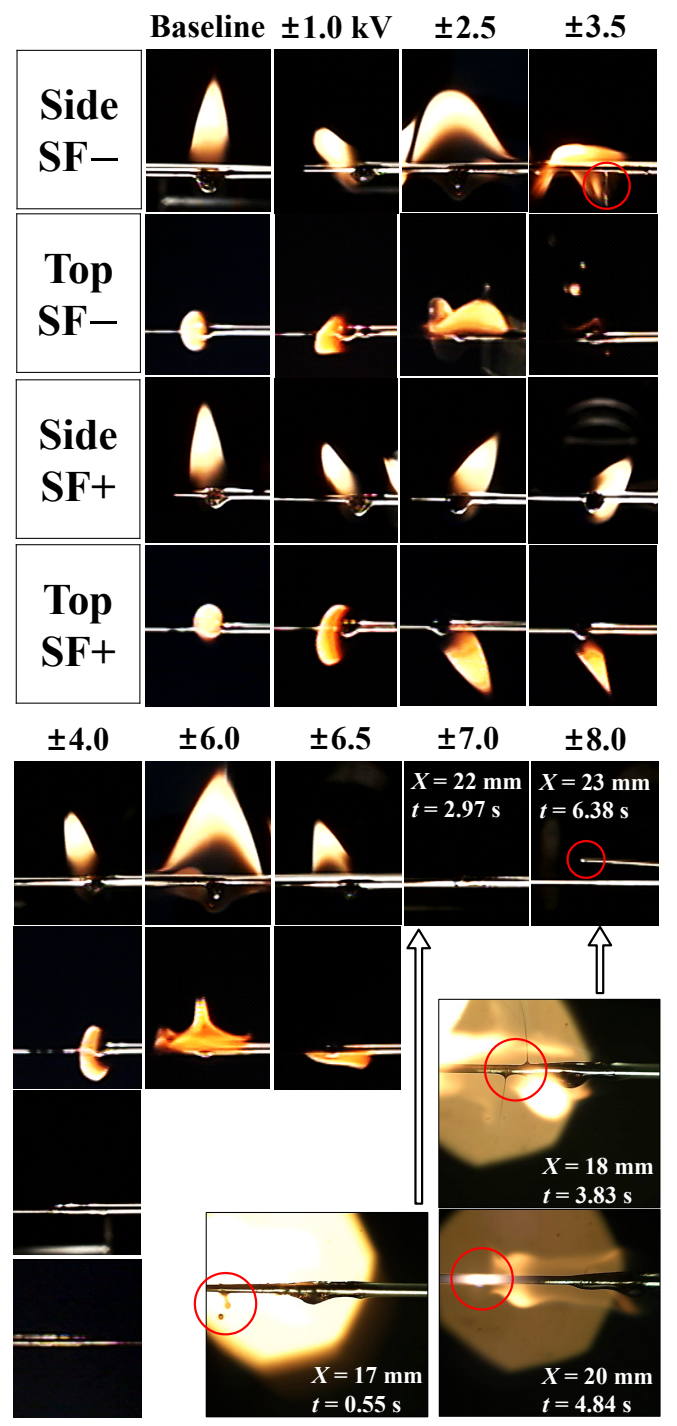

FIGURE 2: Direct photographs of spreading twin flames with varying DC electric fields for $S=51 \mathrm{~mm}$.

For $\pm 4.0 \mathrm{kV}, \mathrm{SF}+$ is extinguished during propagation because the flame is highly stretched toward $\mathrm{SF}-$ due to ionic wind effect while $\mathrm{SF}-$ leans to burnt side again. $\mathrm{SF}-$ spreads up to $\pm 6.5 \mathrm{kV}$ with its size increasing (with the dripping of molten PE at $\pm 6.0 \mathrm{kV}$ ) and then decreasing (with no dripping of molten PE at $\pm 6.5 \mathrm{kV}$ ). SF- leans toward the burned wire at $\pm 6.5 \mathrm{kV}$. For $\pm 7.0 \mathrm{kV}, \mathrm{SF}-$ is extinguished within $22 \mathrm{~mm}$ from the ignition point. The inset image at $0.55 \mathrm{~s}$ shows small molten PE detached continuously from the main body of molten PE due to di-electrophoresis phenomenon [18]. Subsequently fine droplets eject from the detached molten PE due to electrospray [15]. Owing to the mass loss of molten PE, the flame is extinguished at $2.97 \mathrm{~s}$. For $V_{\mathrm{DC}} \geq 8 \mathrm{kV}$, streamers are generated and SF+ is first extinguished due to flame stretch toward SF-. Subsequently SF- experiences excessivee loss of molten PE via electrospray and di-electrophoresis (red circles in inset photos). Then, repetitive generation of streamers between the two wires leads to wire-cutting of SF-.

Three types of flame spreads were identified depending on the wire gap and applied voltage as shown in Fig. 3: both $\mathrm{SF}+$ and $\mathrm{SF}-$ spread with no extinction in regime I; SF+ is extinguished, while SF- keeps on spreading in regime II; $\mathrm{SF}+$ is extinguished and subsequently $\mathrm{SF}-$ is also extinguished in regime III. When $S$ increases, these regimes are extended to higher voltages. Further increase in $V_{\mathrm{DC}}$ from regime III causes the wire for $\mathrm{SF}-$ to be cut by an electrical short.

To show the important role of the interaction between two flames, we first examine the flame front position $\left(X_{\mathrm{f}}\right)$ and the angle $\theta_{1}\left(\theta_{2}\right)$ between the line connecting both flame front positions for SF+ and SF- and the SF+ wire 
(between the tilting direction of SF+ flame toward SF- and the SF+ wire) with time $(t)$ for $S=51 \mathrm{~mm}$ in Fig. 4 for $\pm 2.5 \mathrm{kV}$. Here, "single" denotes single-flame spread (by igniting only one wire) and "twin" means twin-flame spread (by igniting both wires). For the single-flame spread, the flame positions with time are nearly equal irrespective of the positive and negative wires, implying minimal effect of electric field. For twin flame spread, SF- initially spreads faster than $\mathrm{SF}+$ and as time goes on, say after $X_{\mathrm{f}}>165 \mathrm{~mm}$, the two flames spread with equal spread rates having same slopes on $X_{\mathrm{f}}-t$ plot. The results also show that $\theta_{1}\left(\begin{array}{lll}\theta_{2} & \theta \text { becomes }\end{array}\right.$ nearly constant for $X_{\mathrm{f}}>165 \mathrm{~mm}$. These behaviors imply that the

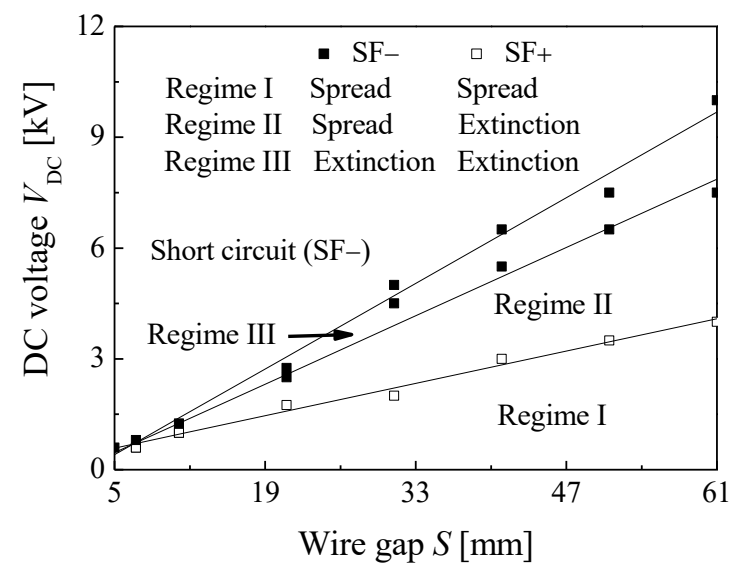

FIGURE 3: Flame stability map in terms of wire gap and voltage.

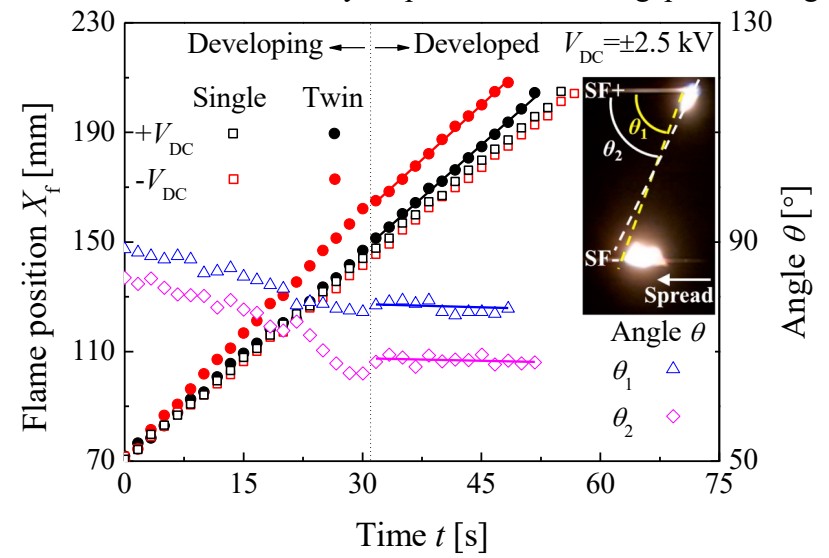

FIGURE 4: Flame front position (Xf), $\theta_{1}$, and $\theta_{2}$ with time in twin flame spreads exhibiting developing and developed regions at $\pm 2.5 \mathrm{kV}$ for $S=51 \mathrm{~mm}$.

twin-flame spread exhibits a transient behavior initially (developing region) and reaches a quasi-steady state (developed region) over a critical flame front position. In all cases, SF- and SF+ spread reasonably linearly with time in the developed regions. Thus, the quasi-steady twin flame spread rate (FSR, $S_{\mathrm{w}}$ ) can be determined from $S_{\mathrm{w}}=d X_{\mathrm{f}} / d t$ in the developed region.

\section{Flame spread rate with applied electric fields}

The FSR for $S=51 \mathrm{~mm}$, shown in Fig. 5, represents overall characteristics. FSR decreases with $V_{\mathrm{DC}}$, increases, and then decreases again until SF+ is extinguished for $V_{\mathrm{DC}}>3.5 \mathrm{kV}$. After the extinction of SF+, SF- corresponds to a single flame propagation where FSR decrease, increases beginning at $5 \mathrm{kV}$ and then decreases again. For the two regimes mentioned above; twin flame spread (regime I) and single flame spread after the extinction of SF+ (regime II), three sub-regimes can be identified as DC voltage increased: regime I-1 and II-1 exhibited a decreasing FSR, regime I-2 and II-2 an increasing FSR, and regime I-3 and II-3 again a decreasing FSR. As the DC voltage further increases, $\mathrm{SF}-$ is also extinguished during propagation. 
As the voltage further increases, the wire for SF- is cut down by short circuit during the transient period due to an excessively strong streamer.

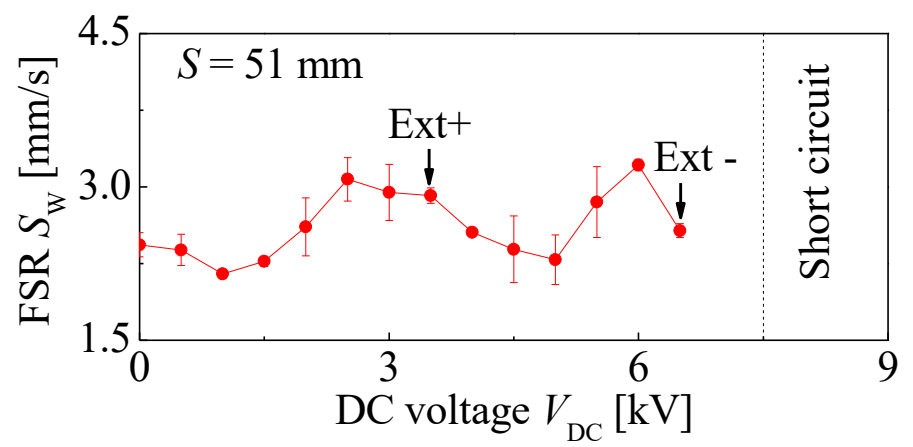

FIGURE 5: Flame spread rates with DC voltage during quasi-steady twin-flame spread and FSRs for single flame.

\section{Flame tilting and dynamic behaviors of molten PE}

Soot particles are formed on the fuel side of a flame, and thereby influenced by the thermophoretic force caused by the temperature gradient between the flame and molten PE or wire along with the electrostatic force, such that particles can be transported inward and deposited onto the molten PE or the wire [13-15]. As shown in Fig. 2, the color of molten PE becomes increasingly dark due to appreciable amount of soot deposition as the voltage increased. This soot deposition could affect the radiation heat transfer, thereby could reduce the surface tension of the molten PE and subsequently lead to dripping of molten $\mathrm{PE}$ at -2.5 and $-6.0 \mathrm{kV}$ for $\mathrm{SF}-$, which is similar to that observed previously for a single wire with applied AC electric fields [13-15].

When the DC voltage was low, the flame leaned toward the bare-wire, e.g., at $1 \mathrm{kV}$ for $S=51 \mathrm{~mm}$ (Fig. 2). The electric field intensity in the bare-wire side is larger than that in the unburned wire side due to the existence of PE insulation, resulting in the leaning of spreading flame toward the bare wire [13-15]. However, because of low voltages applied to the wires, the interaction between the twin flames is very weak such that the twin flames leaning toward the burnt wires spread reasonably independently.

As $V_{\mathrm{DC}}$ increases further, the twin flames start to interact (Fig. 2). Charged particles in a reaction zone can be accelerated by the Lorentz force, resulting in an enhancement of associated diffusion flux. The kinetic energy, gained by charged particles, may also enhance the rate of chemical reaction. Accelerated ions can transfer momentum to neutral particles, generating bulk flow (ionic wind effect). Since positive ions in a flame are abundant than negative ions [12], due to the existence of electrons and charge neutrality, the bulk ionic wind is directed toward the negative potential. Therefore, $\mathrm{SF}+$ is pushed toward the negatively charged wire, making the flame tilted toward the negatively charged wire (e.g., 2.5 and $3.5 \mathrm{kV}$ cases in Fig 2, refer to the top view). In this regard, the flame shape and tilting direction of flame could be affected by ionic wind effect.

The dynamic behaviors of molten PE also influence FSR. The dynamic behavior of spreading flame becomes much more complex when considering them over electrical wire with applied electric fields. Unequal electric field forces between the unburned and burnt wire regions acting on molten PE for the single flame spread over electrical wire with applying AC electric fields forced a droplet of molten PE (a part from main body of molten PE) to move toward the bare wire side having higher electric field intensity. This phenomenon was attributed to the di-electrophoresis [1822].

Sequential images of SF- are demonstrated in Fig. 6 at $\pm 3.5 \mathrm{kV}$ for $S=51 \mathrm{~mm}$. On the main body of molten PE for the negatively charged wire, negative ions/charges drift toward the liquid surface and positive ions drift away from the surface toward the wire. The accumulated negative ions/charges on the surface can be concentrated on the outermost skin layer. When the voltage is sufficiently large, the liquid surface can then be 

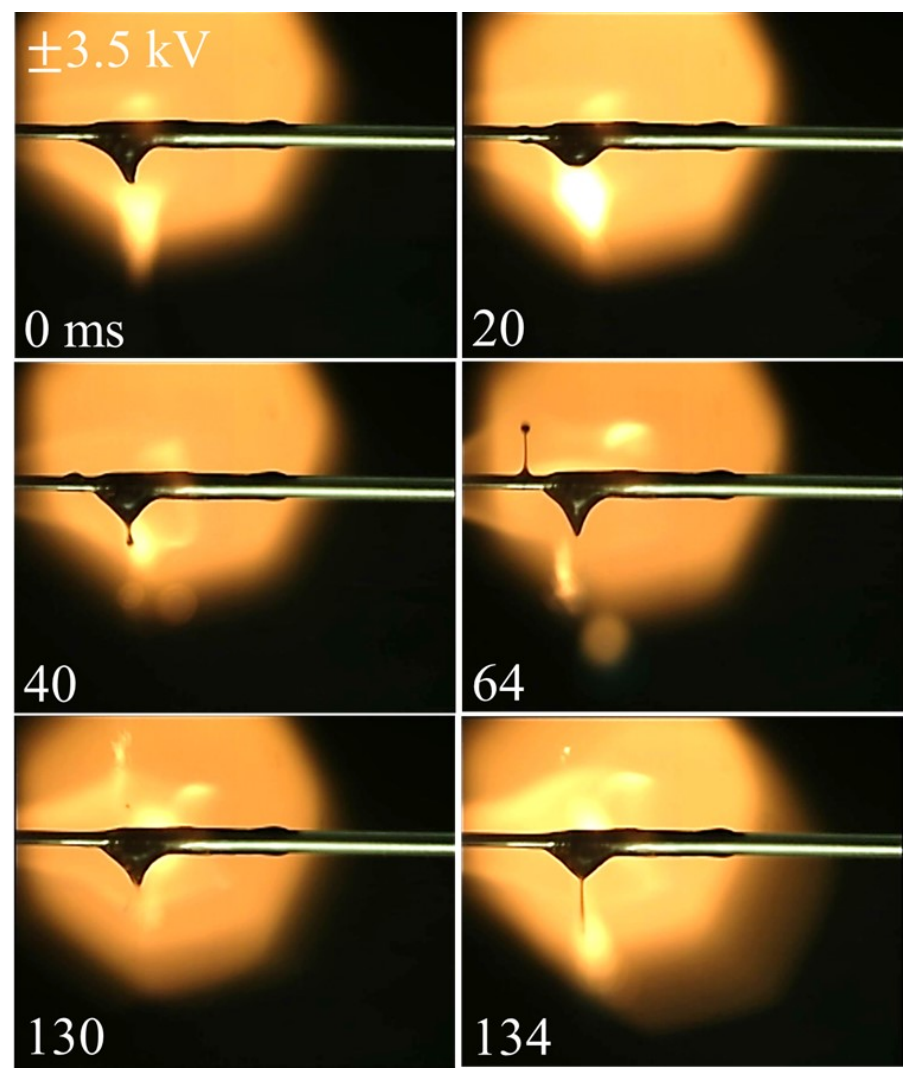

FIGURE 6: Sequential images of SF- in case of simultaneous occurrence of di-electrophoresis and electrospray at

$$
\pm 3.5 \mathrm{kV} \text { for } S=51 \mathrm{~mm} \text {. }
$$

drawn out forming a liquid Taylor cone, and subsequently a series of fine droplets ejected from the surface of molten $\mathrm{PE}$ in the form of an electrospray [23-26], as shown in the photo at $134 \mathrm{~ms}$. This behavior is similar to the previous observations $[15,18]$ for a single-wire case. At 40 and $64 \mathrm{~ms}$, a small droplet is detached from the main body of molten $\mathrm{PE}$ and moved toward the burnt wire due to a di-electrophoresis phenomenon [18]. A Taylor cone on the detached molten PE surface also forms at $64 \mathrm{~ms}$ and an electrospray phenomenon occurred.

Mass loss of molten PE via electrospray and dripping of molten PE can reduce FSR and thereby cause flame extinction while the occurrence of di-electrophoresis phenomenon can increase FSR via increasing effective flame width.

\section{Flame extinction and short circuit}

It was explained that SF+ was pushed toward the negatively charged wire since the bulk ionic wind is directed toward the negative potential. Then, the base of SF+ experiences a blowing effect as a cross-wind toward SF-, leading to a marked stretch effect on $\mathrm{SF}+$ and thereby resulting in the extinction of $\mathrm{SF}+$.

At $7 \mathrm{kV}$ for $S=51 \mathrm{~mm}$ (Fig. 2), a week streamer is generated and then both SF+ and SF- are enlarged momentarily because of the detachment of molten PE from the wire. Simultaneously, SF+ is extinguished before reaching the developed regime because of the stretch toward the $-V_{\mathrm{DC}}$ wire, while $\mathrm{SF}-$ survives with small amount of molten PE and spreads. In this situation, $\mathrm{SF}$ - with a relatively small flame size first experiences appreciable amount of molten $\mathrm{PE}$ detached from the main molten PE due to di-electrophoresis, subsequently experiencing mass loss via electrospray from the detached droplet. The flame experiences mass loss of the main molten PE momentarily due to electrospray phenomenon, subsequently the flame size increases momentarily and then becomes smaller, resultantly restricting the formation of molten PE (while a small droplet is still detached from the main body), thereby reducing flame size further, and leading to flame extinction.

At $8 \mathrm{kV}$ for $\mathrm{S}=51 \mathrm{~mm}$ in Fig. 2, while a strong streamer is generated and then the similar processes observed at 7 $\mathrm{kV}$ are repeated and $\mathrm{SF}+$ extinguishes. Then, a part of bare wire for SF- is overheated by strong streamers. When the overheated part of wire is cut (short circuit), the SF- also extinguishes simultaneously because of the removal of molten PE due to a violent motion of the wire when cutting. 


\section{Conclusion}

The effect of DC electric field on the characteristics of flame spread over polyethylene-insulated twin electrical wires was studied by varying the wire gap and voltage.

Twin flames leaning toward the bare wire spread independently with no interaction when small voltages are applied. When voltage is increased, twin flames start to interact via ionic wind effect. The FSR can be classified into two regimes; twin flame spread and single flame spread after the extinction of SF+. For the twin flame spread case, as voltage increases, the flame leaning toward the burnt wire shows a decreased FSR and, as the two flames interact, FSR increases via the ionic wind effect and then decreases via the mass loss of molten PE due to the electrospray and di-electrophoresis phenomena. The single flame spread case exhibited similar behavior. When the voltage was increased further, the flames were extinguished via streamer generation and an electrical short occurred at excessive voltages.

The results suggest that the fire-safety code developed without considering an applied electric field may need to be modified to consider the situation involving interacting electrical wire fires subject to electric fields.

\section{ACKNOWLEDGEMENTS}

This work was supported by the SGER Program through the National Research Foundation (NRF) of Korea funded by the Ministry of Education, Science, and Technology (2018-2019). SHC and MSC were supported by King Abdullah University of Science and Technology.

\section{REFERENCES}

[1] O. Keski-Rahkonen, J. Mangs, Nuclear Engineering and Design Vol. 213 (2002): pp. 209-221.

[2] A. Matala, S. Hostikka, "Prababilistic simulation of cable performance and water based protection in cable tunnel fires" Nuclear Engineering and Design Vol. 241 No. 12 (2011): pp. 5263-5274.

[3] L. Li, X. Huang, K. Bi, X. Liu, "An enhanced fire hazard assessment model and validation experiments for vertical cable trays" Nuclear Engineering and Design Vol. 301 (2016): pp.32-38.

[4] F. Jia, M. Patel, E. Galea, A. Grandison, J. Ewer, "CFD fire simulation of the swiss air flight 111 in-flight fire part II: Fire spread analysis" Aeronaut. $J$ Vol. 110 No. 1107 (2007): pp. 303-314.

[5] L. Hu, Y. Lu, K. Yoshioka, Y. Zhang, C. Fernandez-Pello, S. H. Chung, O. Fujita, Proc. Combust. Inst. Vol. 36 (2017): pp. 3045-3053.

[6] M. Kikuchi, O. Fujita, K. Ito, A. Sato, T. Sakuraya, Proc. Combust. Inst. Vol. 27 (1998): pp. 2507-2514.

[7] O. Fujita, K. Nishizawa, K. Ito, Proc. Combust. Inst. Vol. 29 (2002): pp. 2545-2552.

[8] A. Umemura, M. Uchida, T. Hirata, J. Sato, Proc. Combust. Inst. Vol. 29 (2002): pp. 2535-2543.

[9] Y. Nakamura, N. Yoshimura, K. Ito, K. Azumaya, O. Fujita, Proc. Combust. Inst. Vol. 32 (2009): pp. 2559-2566.

[10] S. Takahashi, H. Takeuchi, H. Ito, Y. Nakamura, O. Fujita, Proc. Combust. Inst. Vol. 34 (2013): pp. 2657-2664.

[11] NASA STD 6001B, Flammability, offgassing, and compatibility requirements and test procedures, (2011).

[12] J. Lawton, F. J. Weinberg, "Electrical Aspect of Combustion" Clarendon Press, Oxford, (1969).

[13] M.K. Kim, S.H. Chung, O. Fujita, "Effect of AC electric fields on flame spread over electrical wire" Proc. Combust. Inst. Vol. 33 (2011): pp. 1145-1151.

[14] S.J. Lim, M.K. Kim, J. Park, O. Fujita, S.H. Chung, "Flame spread over electrical wire with AC electric fields: Internal circulation, fuel vapor-jet, spread rate acceleration, and molten insulator dripping" Combust. Flame Vol. 162 (2015): pp. 1167-1175.

[15] S.J. Lim, S.H. Park, J. Park, O. Fujita, S.I. Keel, S.H. Chung, "Flame spread over inclined electrical wires with AC electric fields" Combust. Flame Vol. 185 (2017): pp. 82-92.

[16] R. Lanoie, H.P. Mercure, "Influence of forest fire on power line insulation"6th Int. Symp. High Voltage Eng. (1989): pp. 30.06.

[17] W.L. Vosloo, J.P. Holzhausen, A.C. Britten, "Research into the detection of fires under high voltage lines" 7th Int. Symp. High Voltage Eng. (1991): pp. 41.12.

[18] S.H. Park, S.J. Lim, J. Park, S.H. Chung, "Effect of the thickness of polyethylene insulation on flame spread over electrical wire with applied electric field: di-electrophoresis phenomenon", to appear in Combust. Flame (2019).

[19] K. V. I. S. Kaler, R. Prakash, D. Chugh, "Droplet microfluidics for chip-based diagnostics", Biomicrofluidics 4 (2010) 022805 pp.1-17.

[20] T. B. Jones, "Liquid dielectrophoresis on the microscale", J. Electrostatics 51-52 (2001): pp.290-299. 
[21] T. B. Jones, M. Gunji, M. Washizu, M. J. Feldman, "Dielectrophoretic liquid actuation and nanodroplet formation", J Appl Phys 89 (2001): pp.1441-1448.

[22] R. Ahmed, T.B. Jones, "Dispensing picoliter droplets on substrates using dielectrophoresis", J. Electrostatics 64 (2006): pp.543-549.

[23] L. Rayleigh, "On the equilibrium of liquid conducting masses charged with electricity", Phil. Mag. 14(87) (1882): pp.184-186.

[24] A. Gomez, K. Tang, "Charge and fission of droplets in electrostatic sprays", Phys. Fluids 6(1) (1994): pp.404414.

[25] G. Taylor, "Disintegration of water droplets in an electric field", Proc. Royal Soc. London A: Mathematical, Physical \& Engineering Sciences 291 (1965): pp.145-158.

[26] S.P. Lin, G. Roberts, "Waves in a viscous liquid curtain", J. Fluid Mech. 112 (1981): pp.443-458. 